\title{
Photoproduction of mesons and Compton scattering on the proton: Selected results from the $\mathrm{A} 2$ Collaboration at MAMI
}

\author{
Vahe Sokhoyan for the A2 Collaboration at MAMI
}

${ }^{1}$ University of Mainz, Institute for Nuclear Physics

\begin{abstract}
The A2 Collaboration performs a manifold research program using real photons in the Crystal Ball/TAPS experiment at the MAMI accelerator facility in Mainz. The experiments take advantage of highintensity unpolarized, linearly or circularly polarized photon beams, and unpolarized or polarized targets. The detector setup provides almost complete coverage in solid angle and is well suited for the detection of multiparticle final states. In order to probe the internal structure of the nucleon, the spectrum of baryon resonances is studied via measurements of unpolarized cross sections and various polarization observables in single and double meson photoproduction. The program aiming to determine the scalar and spin polarizabilities of the nucleons with high precision is performed with the Compton scattering experiments. In 2017, the focal plane detector used in the tagging system of the Crystal Ball/TAPS experiment was completely renewed, allowing new measurements with unprecedentedly high precision. This paper presents recent selected results from the A2 Collaboration at MAMI.
\end{abstract}

\section{Introduction}

The program carried out by the A2 Collaboration covers a broad range of topics in hadron physics. The experiments are performed with various combinations of unpolarized, linearly or circularly polarized tagged photons with unpolarized or polarized targets. The polarization of recoil nucleons can be measured with an available recoil polarimeter. In addition to the proton targets, various experiments are carried out with light and heavy nuclei for the studies of mesons photoproduction on neutrons and search for inmedium modifications of hadrons in the nuclear medium. The experimental setup consisting of the Crystal Ball and TAPS calorimeters provides a nearly $4 \pi$ acceptance coverage.

At energies below pion threshold (80-140 MeV), new data sets on Compton scattering on the proton were recently acquired. Based on these data sets, the scalar polarizabilities of the proton will be extracted with an unprecedented precision. Moreover, the beam asymmetry $\Sigma_{3}$ was measured for Compton scattering below pion threshold for the first time, in order to obtain complementary information for the extraction of the proton scalar polarizabilities, in addition to the unpolarized cross section.

At higher energies, various measurements are performed for single and double meson photoproduction in order to study the dynamics and properties of baryon resonances. The study of double meson final states is mainly focused on photoproduction of $2 \pi^{0}$ and $\pi^{0} \eta$ pairs. Recently, we obtained a new data set for differential cross sections and beam helicity asymmetry for the $\gamma p \rightarrow$

*e-mail: sokhoyan@uni-mainz.de $p \pi^{0} \eta$ reaction at photon energies from the threshold up to $1.45 \mathrm{GeV}$. Moreover, the beam helicity asymmetry was measured for the first time for the photoproduction of $\pi^{0} \eta$ pairs on heavy nuclei.

This paper includes recent results from the proton scalar polarizability measurement and the new results on the production of $\pi^{0} \eta$ pairs on a free proton and nuclear targets.

\section{Experimental Setup}

The experiments were performed with a tagged photon beam at the MAMI accelerator in Mainz $[1,2]$. The electron beam impinged on an amorphous or a diamond radiator, the electrons were deflected in the magnetic field and detected by the focal plane detector [3-5]. The recent upgrade of the focal plane detector with new plastic scintillators, equipped with Si-PMTs significantly extended the capability of the tagging system in handling high rates. The Crystal Ball/TAPS calorimeter system, used to detect particles in the final state is shown schematically in Fig. 1. The Crystal Ball calorimeter covers polar angles from $20^{\circ}$ to $160^{\circ}$ and consists of $672 \mathrm{NaI}$ crystals [6], whereas TAPS covers the forward angles $\left(1^{\circ}-20^{\circ}\right)$ and is built of $366 \mathrm{BaF}_{2}$ and $72 \mathrm{PbWO}_{4}$ crystals [7, 8]. Both calorimeters have a full coverage in azimuthal angle. The Crystal Ball calorimeter is additionally equipped with a Particle Identification Detector (PID), made of 24 scintillator bars [9] and with two Multiwire Proportional Chambers [10], which are used for identification and tracking of charged particles. In the forward region, covered by the TAPS calorimeter, plastic veto scintillators are used to distinguish between charged and neutral particles. 


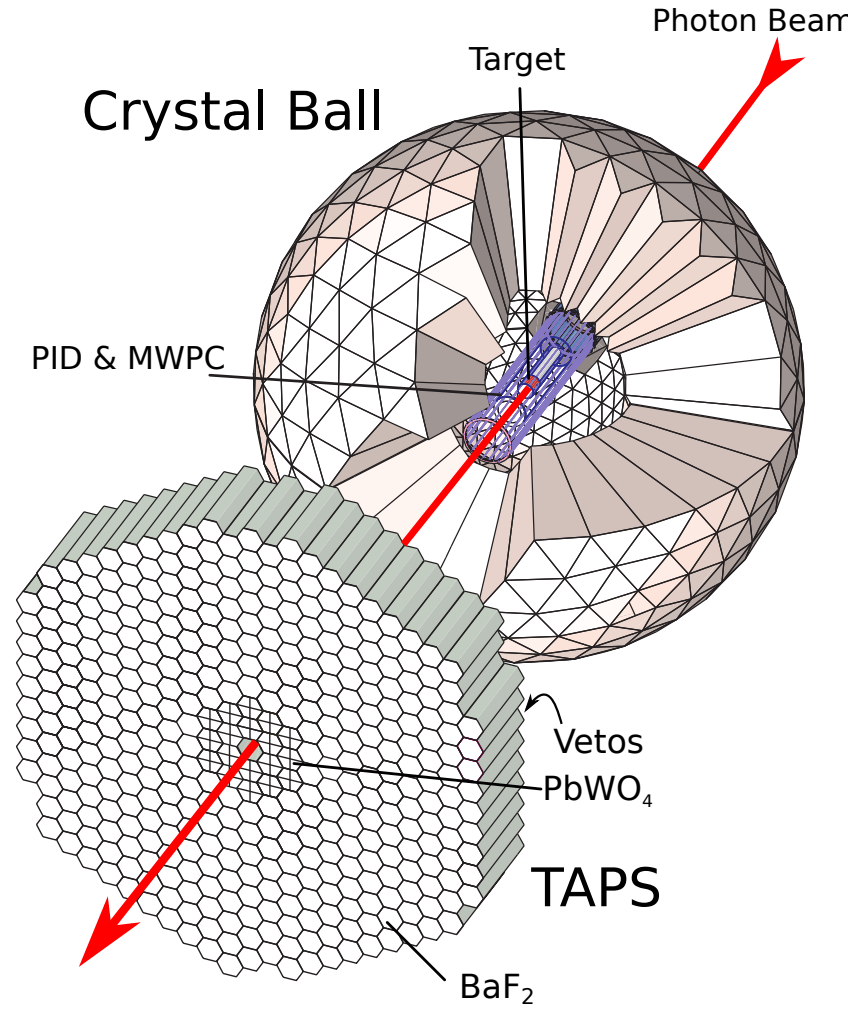

Figure 1. Schematic view of the Crystal Ball/TAPS detection system.

\section{Measurement of the proton scalar polarizabilities}

The scalar polarizabilities are fundamental properties of the proton. They are of great importance for nuclear and atomic physics, and other related fields. Moreover, these polarizabilities are currently a significant source of uncertainty in the determination of the proton charge radius from muonic hydrogen Lamb shift [11]. Additionally, the precise determination of the scalar polarizabilities is crucial for the extraction of the spin polarizabilities. Previously, the proton scalar polarizabilites were extracted from the unpolarized cross section of Compton scattering on the proton at low energies (see, e.g. Refs. [12-15]). The largest of the data sets for Compton scattering was previously obtained with the TAPS setup at MAMI [12].

Recently, we acquired new data sets on Compton scattering for the photon energy range $80-140 \mathrm{MeV}$, measured the unpolarized cross section for Compton scattering with unprecedented precision and performed the first ever measurement of the beam asymmetry $\Sigma_{3}$ below pion production threshold. As discussed in Ref. [16], the measurement of the beam asymmetry provides a potentially complementary sensitivity to the scalar polarizabilities of the proton in addition to the measurement of the unpolarized cross section.

Experimentally, the beam asymmetry $\Sigma_{3}$ is measured with linearly polarized photon beam and unpolarized tar- get and is defined as:

$$
\Sigma_{3} \equiv \frac{\sigma_{\|}-\sigma_{\perp}}{\sigma_{\|}+\sigma_{\perp}}
$$

where the cross sections $\sigma_{\|}$and $\sigma_{\perp}$ are extracted with the photon polarization being either parallel or perpendicular to the scattering plane.

The results of the first pilot experiment with the Crystal Ball/TAPS setup [17] proved the feasibility of a highprecision measurement of the proton scalar polarizabilities $\alpha_{E 1}$ and $\beta_{M 1}$ with the A2 setup. Figure 2 shows an example missing mass distribution from the pilot experiment (the scattered photon was detected in the Crystal Ball). The signal is clearly identified and the shape of the experimental spectra is in good agreement with the distribution obtained from Monte Carlo simulation, indicating a low background contamination.

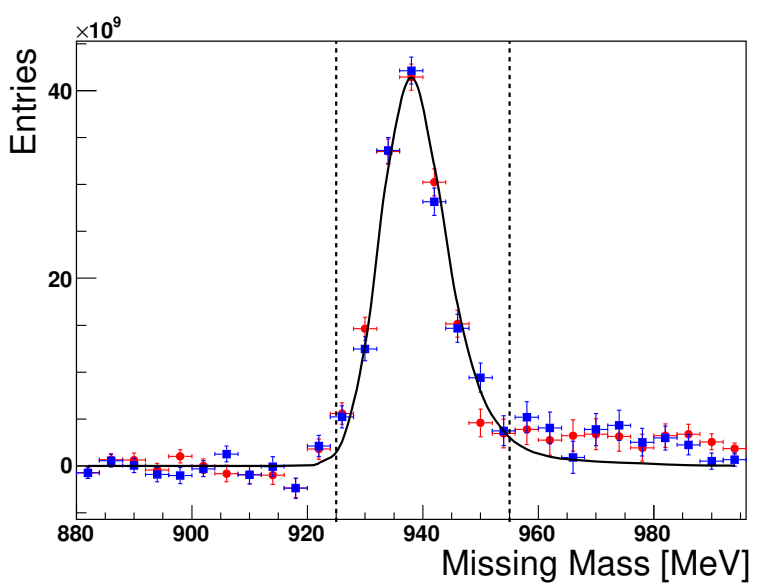

Figure 2. Compton scattering on the proton: Missing mass for the incoming photon energy range 79-98 MeV and polar angles for the scattered photon covering the range $30^{\circ}<\theta<155^{\circ}$. The data were taken with linearly polarized photons with polarization planes either parallel (red circles) or perpendicular to the horizontal plane (blue squares). The black curve shows the corresponding distribution obtained from our Monte Carlo simulation. The dashed lines indicate the cut on the missing mass applied in the analysis. Figure taken from Ref. [17].

After the upgrade of the tagging system, new data were taken by the A2 Collaboration in 2018 with a linearly polarized photon beam and an unpolarized liquid hydrogen target and the analysis is underway $(\mathrm{PhD}$ of $\mathrm{E}$. Mornacchi, Mainz) $[18,19]$. The new data set shows an improvement of about six times in statistics compared to the pilot experiment. In total, more than $1.2 \times 10^{6}$ Compton scattering events were acquired below pion photoproduction threshold in the range $E_{\gamma}=80-140 \mathrm{MeV}$, constituting by far the highest statistics data set compared to all previous measurements.

Figures 3 and 4 show preliminary results for the unpolarized cross section and beam asymmetry $\Sigma_{3}$, respectively. Comparison of the new cross section data [18, 19] with the highest statistics data set available till now — the 


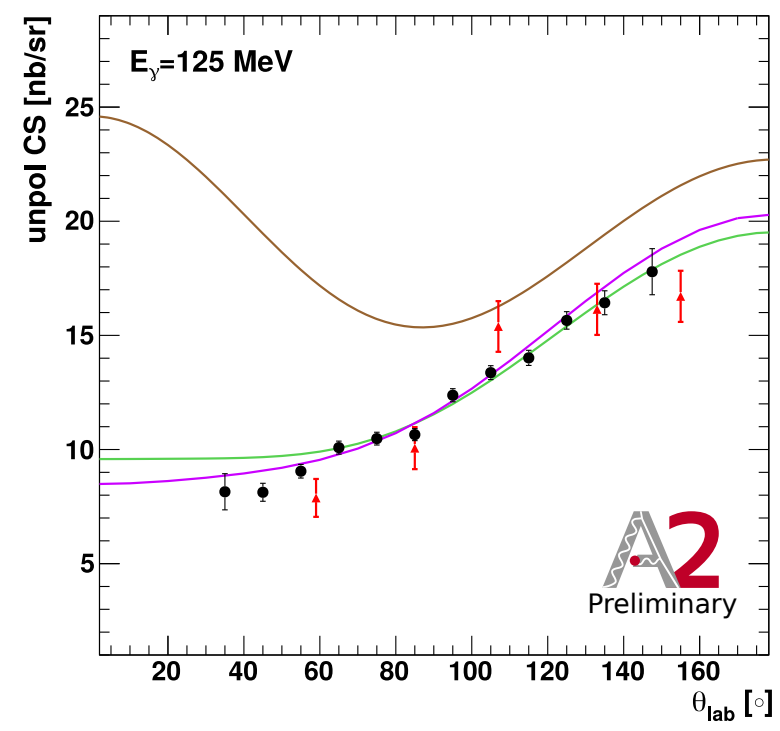

Figure 3. Preliminary unpolarized cross section (black circles) [18], compared to the previous results from Ref. [12] (red triangles). The curves correspond to the Born contribution (brown), Dispersion Relation calculation (magenta) [20, 21], and heavy baryon ChPT calculation (green) [22]. The Dispersion Relation and heavy baryon $\mathrm{ChPT}$ calculations were performed for $\alpha_{E 1}=10.65 \times 10^{-4} \mathrm{fm}^{3}$ and $\beta_{M 1}=3.15 \times 10^{-4} \mathrm{fm}^{3}$.

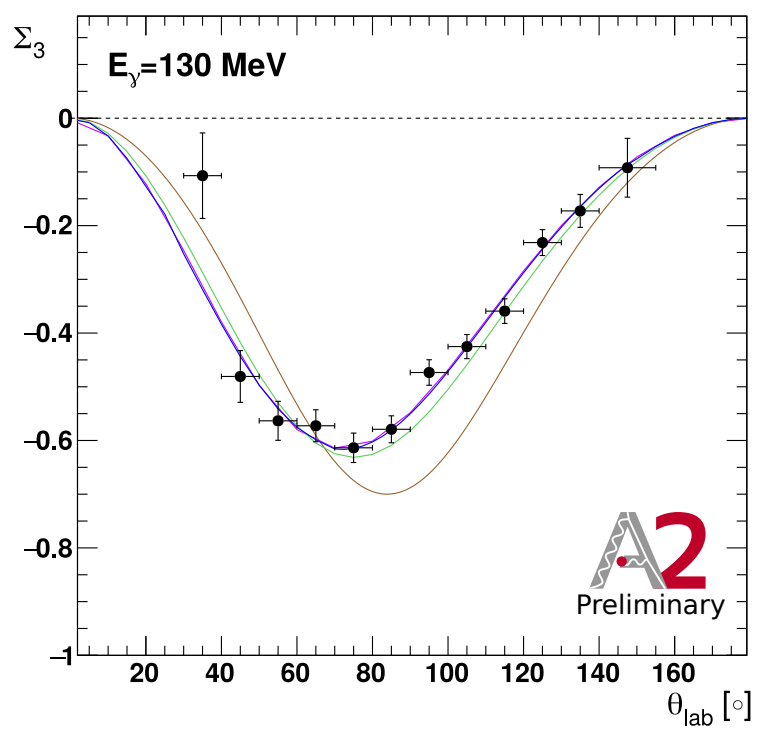

Figure 4. Preliminary beam asymmetry $\Sigma_{3}$ (black circles) [18]. The curves correspond to the Born contribution (brown), Dispersion Relation calculation (magenta) [20, 21], heavy baryon ChPT calculation (green) [22], and ChPT (blue) [23]. All theoretical calculations were performed for $\alpha_{E 1}=10.65 \times 10^{-4} \mathrm{fm}^{3}$ and $\beta_{M 1}=3.15 \times 10^{-4} \mathrm{fm}^{3}$.
MAMI 2001 data [12] — indicates the very significant improvement in statistical accuracy. Moreover, the new data allow us to extend the angular coverage to smaller scattered photon angles. The beam asymmetry $\Sigma_{3}$ from the new measurement is in good agreement with the theoretical calculations. The systematic accuracy is improved compared to the pilot experiment [17] due to the upgrades made to the experimental apparatus, including a more stable linear polarization, improved efficiency of the new tagging system, continuous monitoring of the photon flux, and enhanced performance of the refurbished data acquisition system.

\section{Photoproduction of $\pi^{0} \eta$ pairs on the proton}

Within the last years, a significant progress has been made at different experiments studying the production of $\pi^{0} \eta$ pairs on the proton and [24-31] and light nuclei (deuteron and helium) $[32,33]$. At energies below $1.5 \mathrm{GeV}$, the $D_{33}$ wave plays an important role. In the region of the threshold the contribution of the $\Delta(1700) 3 / 2^{-}$resonance dominates, whereas the importance of the $\Delta(1940) 3 / 2^{-}$resonance increases at higher energies [24-29, 34-36]. Thus, the production of $\pi^{0} \eta$ pairs is well-suited for studying the $\Delta(1700) 3 / 2^{-}$resonance at low energies without being strongly affected by other contributions. Furthermore, an additional selectivity in transitions between decays $N^{*}$ or two $\Delta^{*}$ resonances is introduced due to the fact that the $\eta$ meson acts as an isospin filter.

In 2018, we published a new data set for the reaction $\gamma p \rightarrow p \pi^{0} \eta$ covering photon energies from the threshold up to $\mathrm{E}_{\gamma}=1.45 \mathrm{GeV}$ [37]. Figures 5 and 6 show the new differential cross section for the reaction $\gamma p \rightarrow p \pi^{0} \eta$ compared with previously existing data and model calculations. Our new data agree well with results from the previous measurements with the A2 [24] and CBELSA/TAPS experiments [29]. The new data set is described reasonably by the new version of the Mainz model [37] and Bonn-Gatchina Partial Wave Analysis (BnGa PWA) [29]. Some discrepancies present at low and high energies (see Ref. [37] for further details) indicate potential impact of the data for model calculations. After fitting the new data within the framework of the Mainz model, the parameters of the dominant $\Delta(1700) 3 / 2^{-}$resonance remained practically the same as in the previous version of the Mainz model. However, some changes were obtained for other resonances (e.g. for $\Delta(1940) 3 / 2^{-}$). Further details on the influence of the new data on the resonance parameters can be found in Ref. [37]. In the future, the new data can be added to partial wave analyses on an event-by-event basis using available 4-vectors.

\section{Production of $\pi^{0} \eta$ pairs on carbon, aluminum and lead}

Due to the selectivity in terms of contributions of baryon resonances and their decays, photoproduction of $\pi^{0} \eta$ pairs on nuclei is well suited for studying possible modifications 

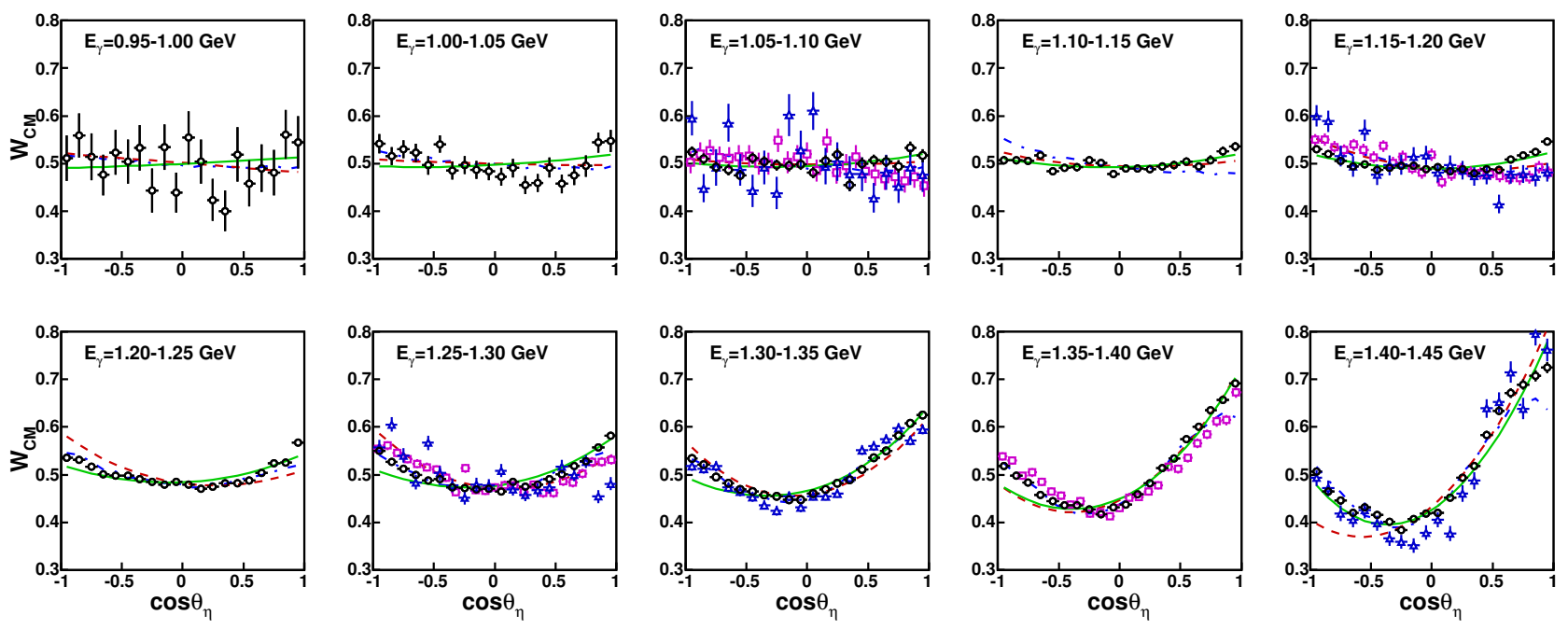

Figure 5. Angular distributions $W_{C M}\left(\theta_{\eta}\right)$ (normalized to unity) for the reaction $\gamma p \rightarrow p \pi^{0} \eta$ : New A2 data (open circles), data from CBELSA/TAPS [27, 29] (blue stars, combination of results from both papers), and previous A2 data [24, 35] (magenta open squares) at energies close to the ones from the new data set. Theoretical curves: Predictions of the BnGa PWA [29] (blue dash-dotted curve), previous version of the Mainz model [26] (red dashed curve), and the new version of the Mainz model where the new A2 data were included (solid green curve). Figure taken from Ref. [37].
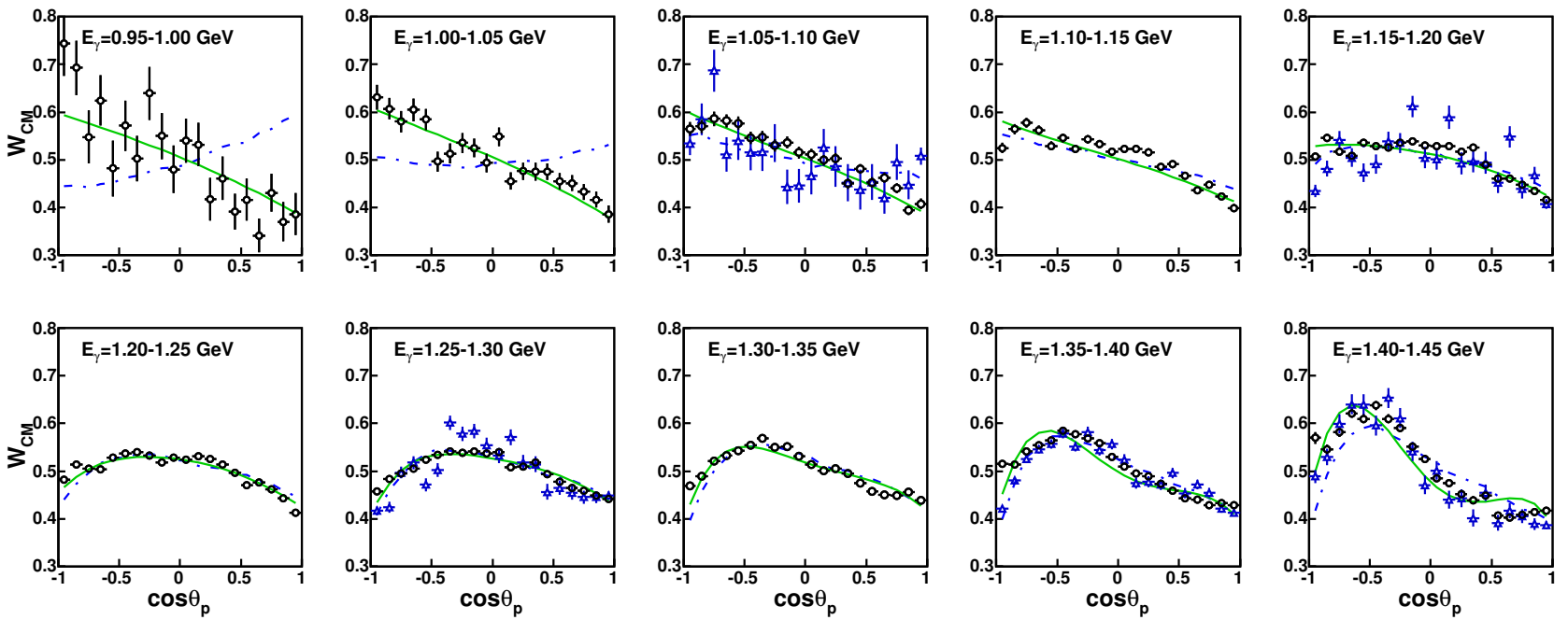

Figure 6. The same notation as for Fig. 5, but the angular distributions for the proton are shown. Figure taken from Ref. [37].

of the contributing resonances in the nuclear medium. The previous results obtained for the proton target $[24,25,27-$ $31,37]$ and recent results for the deuteron from the A2 collaboration $[32,33]$ confirmed the dominance of the $D_{33}$ partial wave for the production $\pi^{0} \eta$ pairs on the proton and neutron. Recently, we obtained new data on the photoproduction of $\pi^{0} \eta$ pairs for carbon, aluminum, and lead targets and measured the beam helicity asymmetry $I^{\odot}$ for these nuclei for the first time [38]. These measurements allowed testing whether the contribution of the $D_{33}$ partial wave still dominates the $\pi^{0} \eta$ photoproduction mechanism on heavy nuclei. As shown previously for the deuteron target [32], the beam helicity asymmetry is very similar for the free proton and deuteron target, although the unpolarized cross section is significantly reduced. Therefore, one could assume that the beam helicity asymmetry used for studying the production of the $D_{33}$ partial wave on heavy nuclei would not be affected significantly by the Final State Interaction (FSI) effects.

The asymmetry $I^{\odot}$, originating due to the contribution of the $D_{33}$ wave can be expressed [25]:

$$
I^{\odot}\left(\Phi_{\pi}\right)=A_{1} \sin \Phi_{\pi}+A_{2} \sin 2 \Phi_{\pi},
$$

where the angle $\Phi_{\pi}$, is defined as the azimuthal pion angle in the $\pi N$ rest frame with respect to the plane determined by the momenta of the incident photon and $\pi N$ system in the center-of-mass (c.m.) frame.

Due to this feature, the observable $I^{\odot}$ can be used to study the modifications of the amplitudes in the nuclear environment and compared to the production on a free nucleon. Beyond conventional (FSI) effects, the most likely 

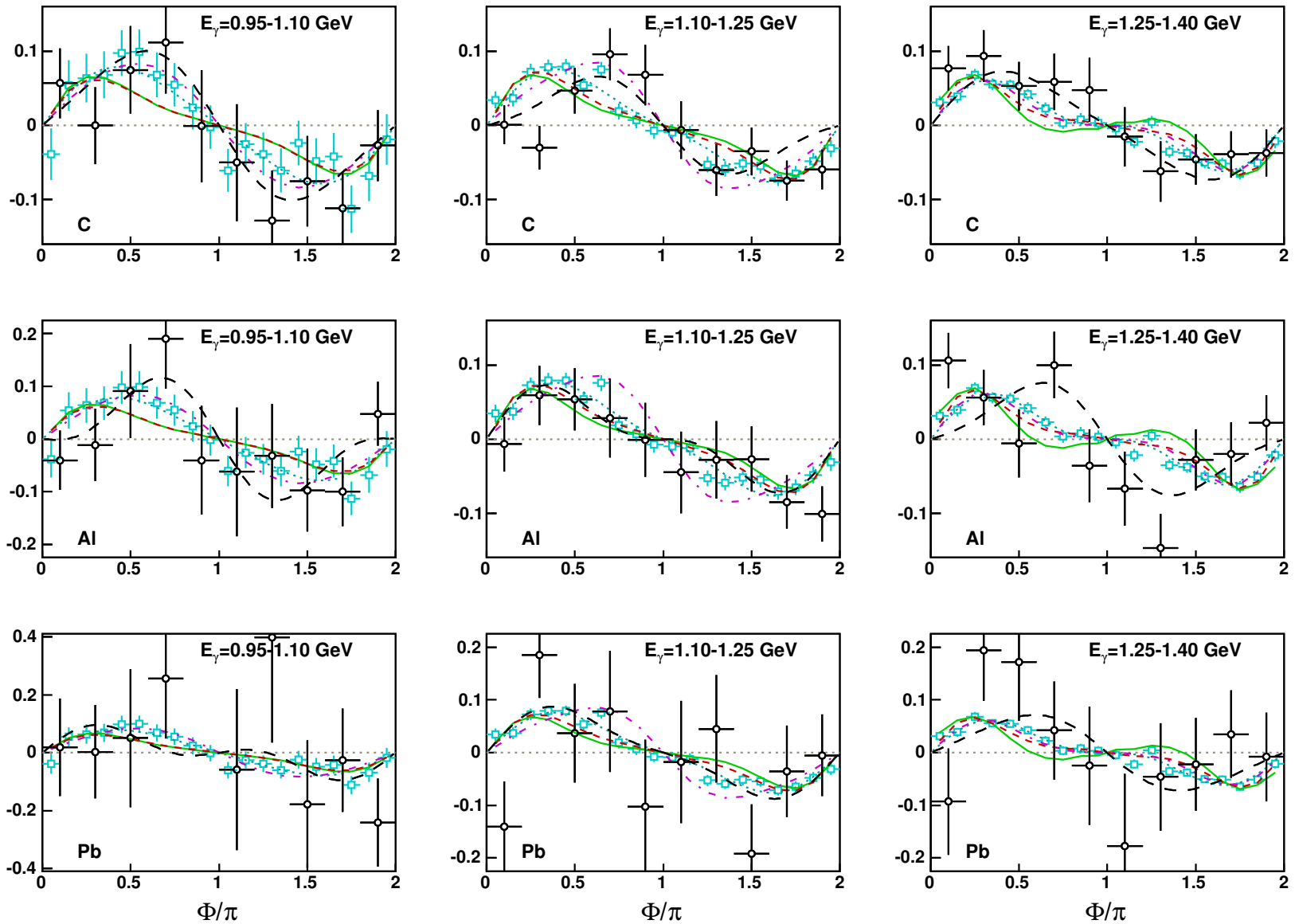

Figure 7. Beam-helicity asymmetry $I^{\odot}$ for the carbon, aluminum, and lead targets (open circles) and the free proton data (cyan open squares) [37]. Curves: Previous version of the Mainz model [25] (magenta dash-dotted line), latest version of the Mainz model from Ref. [37] for a free proton (red dashed line) and for the carbon, aluminum, or lead targets (solid green line). Fits to the experimental data points are performed according to function from Eq. (2) for the nuclear targets (long-dashed black line) and for the hydrogen target (cyan dotted line). The results for carbon, aluminum, and lead are shown in top, middle, and bottom rows, respectively. The data point in the bin $0.8<\Phi / \pi<1.0$ and $0.95<E_{\gamma}<1.10 \mathrm{GeV}$ for $\mathrm{Pb}$ is at $I^{\odot}=-0.65 \pm 0.30$. Figure taken from Ref. [38].

modifications in the nuclear medium could be the suppression or an increase of the width of the $D_{33}$ states. Due to the inelastic mechanisms, the relative contributions of contributing terms can be modified, leading to modifications of the shape of the observable $I^{\odot}\left(\Phi_{\pi}\right)$ described by Eq. (2).

In the new experiment, we measured the observable $I^{\odot}\left(\Phi_{\pi}\right)$ for carbon, aluminum, and lead targets. The beam helicity asymmetry is measured using circularly polarized photons and unpolarized target and can be expressed as:

$$
I^{\odot}(\Phi)=\frac{d \sigma^{+}-d \sigma^{-}}{d \sigma^{+}+d \sigma^{-}}=\frac{1}{P_{\gamma}} \frac{N^{+}-N^{-}}{N^{+}+N^{-}}
$$

where $d \sigma^{ \pm}$corresponds to the differential cross sections as a function of angle $\Phi$ (denoted as $\Phi_{\pi}$ in our case) for the two helicity states of the incoming photon, $P_{\gamma}$ is the degree of circular polarization of the photon, and $N^{ \pm}$represents intensities for each of the two helicity states at the angle $\Phi$.

The beam helicity asymmetry was extracted from events with four or five photon candidates in the final state (for further analysis details see Ref. [38]). The $\pi^{0} \eta$ events corresponding to the production on protons and neutrons were not separated in the analysis because it was not possible to distinguish between these channels for four-cluster events. Also, it was assumed in the analysis that the target particle had nucleon mass and zero momentum.

Figure 7 shows the beam helicity asymmetry $I^{\odot}(\Phi)$ for the obtained $\gamma N \rightarrow 4 \gamma X$ events for nuclear targets in comparison with the previous A2 measurement on a free proton [37]. The experimental data for the nuclear targets are generally in good agreement with the free proton data. The data are also compared with the calculations performed within the previous [25] and the latest [37] versions of the Mainz model. The calculations shown for the Mainz model for the nuclear targets are described in Ref. [38] and include FSI effects, showing very similar behavior for the nuclear targets and free proton. Moreover, the model calculations are in agreement with the data obtained with nuclear and free proton targets.

These comparisons are demonstrated in a more quantitative way in Fig. 8, where shown are the coefficients $A_{1}$ and $A_{2}$ from Eq. (2), extracted from fitting the $I^{\odot}\left(\Phi_{\pi}\right)$ distributions with a function from Eq. (2). These coefficients obtained for the nuclear targets are compared to each other, 
to the values obtained for the free proton, and to the corresponding predictions of the Mainz model. The coefficients $A_{1}$, corresponding to the contribution of the $D_{33}$ wave only, are in good agreement for the nuclear targets and free proton (both for the data and model calculations). The coefficients $A_{2}$, describing the interference of the $D_{33}$ wave with other waves, are significantly smaller than $A_{1}$, again showing a reasonable agreement between nuclear targets and for free proton data within the statistical errors.
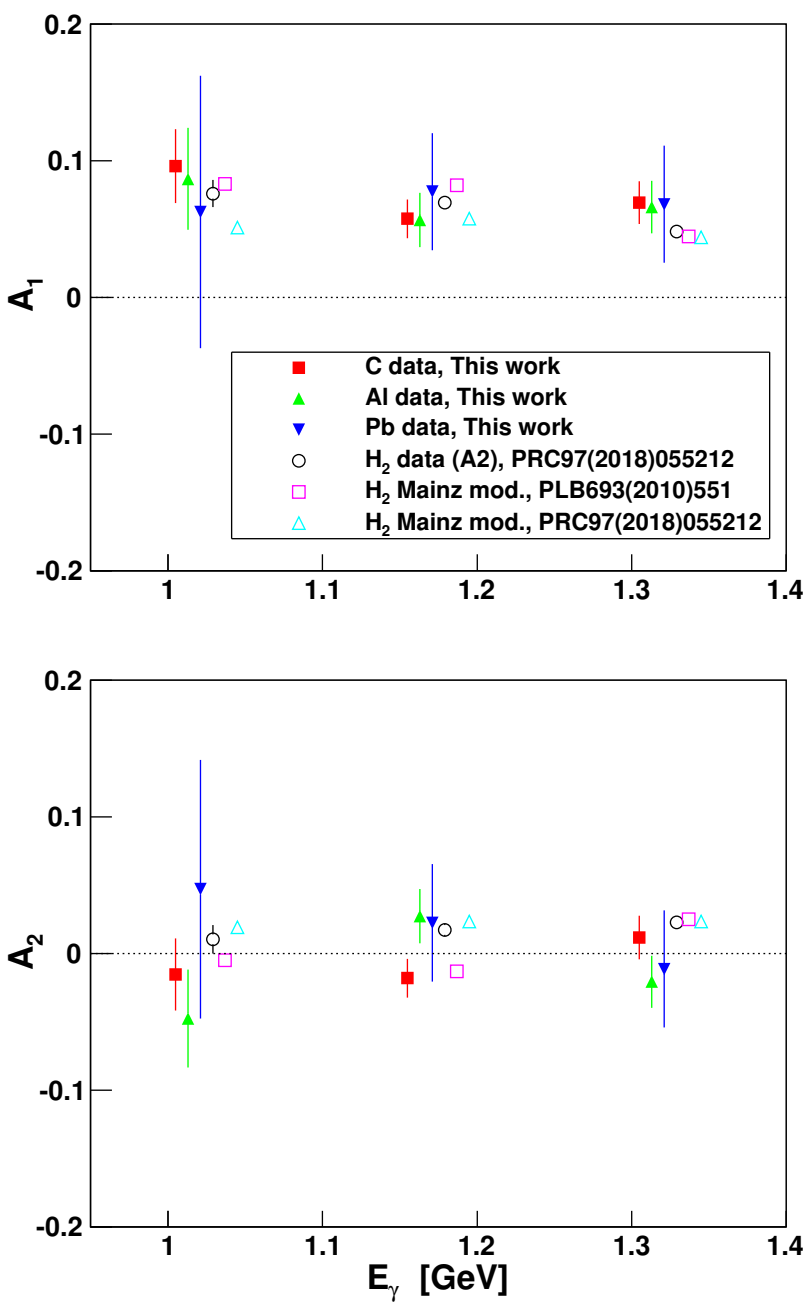

Figure 8. Coefficients $A_{1}$ and $A_{2}$ obtained from fitting $I^{\odot}(\Phi)$ distributions with a function from Eq. (2). Shown are the coefficients obtained for the nuclear targets, free proton data [37], and results from the previous [25] and the latest Mainz model [37]. Figure taken from Ref. [38].

The good agreement for $I^{\odot}(\Phi)$ measured for the nuclear targets and with the free proton indicates similar mechanisms for the photoproduction of $\pi^{0} \eta$ pairs (dominated by contribution of the $D_{33}$ wave in our energy range). Interestingly, this observation is consistent with the measurements performed earlier by the A2 Collaboration with a deuterium target [32], where the results for $I^{\odot}(\Phi)$ on a quasi-free proton and neutron were found to be in agreement with the free-proton data. In analogy with the deuteron results, our new data indicate that the asym- metry $I^{\odot}(\Phi)$ is not significantly affected by FSI effects for heavier nuclear targets.

The obtained results stimulate further searches for inmedium modifications of baryon resonances by measuring polarization observables in general and observable $I^{\odot}(\Phi)$ in particular. Moreover, this study represents the first measurement of the production of $\pi^{0} \eta$ pairs on heavy nuclei, opening a route for further studies of this kind.

\section{References}

[1] H. Herminghaus et al., IEEE Trans. Nucl. Sci. 30, 3274 (1983).

[2] K.-H. Kaiser et al., Nucl. Instrum. Methods Phys. Res. A 593, 159 (2008).

[3] I. Anthony et al., Nucl. Instrum. Methods Phys. Res. A 301, 230 (1991).

[4] S. J. Hall et al., Nucl. Instrum. Methods Phys. Res. A 368, 698 (1996).

[5] J. C. McGeorge et al., Eur. Phys. J. A 37, 129 (2008).

[6] A. Starostin et al., Phys. Rev. C 64, 055205 (2001).

[7] R. Novotny, IEEE Trans. Nucl. Sci. 38, 379 (1991).

[8] A. R. Gabler et al., Nucl. Instrum. Methods Phys. Res. A 346, 168 (1994).

[9] D. Watts et al., Chin. Phys. C 33, 1183-1188 (2009).

[10] D. Watts, The Crystal Ball and TAPS detectors at the MAMI electron beam facility, in Proceedings of the 11th nternational Conference on Calorimetry in Particle Physics (Color2004), Perugia, Italy 2004 (World Scienti c, 2005) (World Scientific, 2004), Vol. 560.

[11] A. Antognini et al., Science 339, 417 (2013).

[12] V. Olmos de Leon et al., Eur. Phys. J. A 10, 2072013215 (2001).

[13] F. J. Federspiel et al., Phys. Rev. Lett. 67, 1511 (1991).

[14] B. E. MacGibbon et al., Phys. Rev. C 52, 2097 (1995).

[15] A. Zieger et al., Phys. Lett. B 278, 34 (1992).

[16] N. Krupina, V. Pascalutsa, Phys.Rev.Lett. 110, 262001 (2013).

[17] V. Sokhoyan et al., Eur. Phys. J. A 53, 14 (2017).

[18] E. Mornacchi (University of Mainz), PhD thesis in preparation.

[19] E. Mornacchi [A2 Collaboration], EPJ Web Conf. 199, 05020 (2019).

[20] D. Drechsel, M. Gorchtein, B. Pasquini, M. Vanderhaeghen, Phys. Rev. C 61, 015204 (1999).

[21] B. Pasquini, D. Drechsel, M. Vanderhaeghen, Phys. Rev. C 76, 015203 (2007).

[22] J.A. McGovern, D.R. Philips, H.W. Grießhammer, Eur. Phys. J. A 490, 12 (2013).

[23] V. Lensky and V. Pascalutsa, Eur. Phys. J. C 65, 195 (2010).

[24] V. L. Kashevarov et al., Eur. Phys. J. A 42, 141 (2009).

[25] V. L. Kashevarov et al., Phys. Lett. B 693, 551 (2010). 
[26] A. Fix, V. L. Kashevarov, A. Lee, and M. Ostrick, Phys. Rev. C 82, 035207 (2010).

[27] I. Horn et al., Eur. Phys. J. A 38, 173 (2008).

[28] E. Gutz et al., Phys. Lett. B 687, 11 (2010).

[29] E. Gutz et al., Eur. Phys. J. A 50, 74 (2014).

[30] J. Ajaka et al., Phys. Rev. Lett. 100, 052003 (2008).

[31] T. Nakabayashi et al., Phys. Rev. C 74, 035202 (2006).

[32] A. Käser et al., Eur. Phys. J. A 52, 272 (2016).
[33] A. Käser et al., Phys. Lett. B 786, 305 (2018).

[34] M. Döring, E. Oset, and D. Strottman, Phys. Rev. C 73, 045209 (2006).

[35] A. Fix, M. Ostrick, and L. Tiator, Eur. Phys. J. A 36, 61 (2008).

[36] A. Fix, V.L. Kashevarov, M. Ostrick, NP A 909, 1 (2013).

[37] V. Sokhoyan et al., Phys. Rev. C 97, 055212 (2018).

[38] V. Sokhoyan et al., Phys. Lett. B 802, 135243 (2020). 